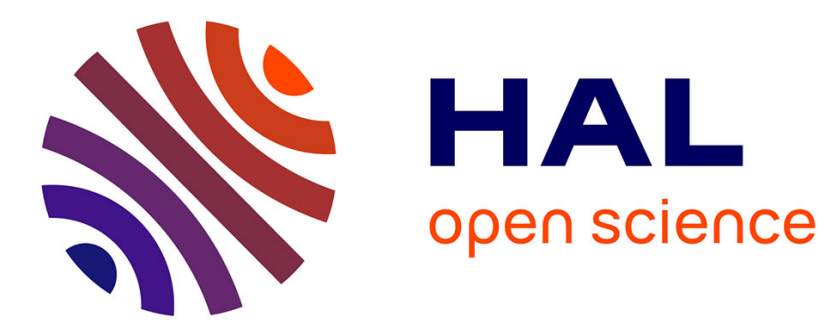

\title{
ECG Fiducial Point Extraction Using Switching Kalman Filter
}

Mahsa Akhbari, Nasim Montazeri Ghahjaverestan, Mohammad B. Shamsollahi, Christian Jutten

\section{- To cite this version:}

Mahsa Akhbari, Nasim Montazeri Ghahjaverestan, Mohammad B. Shamsollahi, Christian Jutten. ECG Fiducial Point Extraction Using Switching Kalman Filter. Computer Methods and Programs in Biomedicine, 2018, 157 (April), pp.129-136. 10.1016/j.cmpb.2018.01.018 . hal-01713406

\section{HAL Id: hal-01713406 \\ https://hal.science/hal-01713406}

Submitted on 20 Feb 2018

HAL is a multi-disciplinary open access archive for the deposit and dissemination of scientific research documents, whether they are published or not. The documents may come from teaching and research institutions in France or abroad, or from public or private research centers.
L'archive ouverte pluridisciplinaire HAL, est destinée au dépôt et à la diffusion de documents scientifiques de niveau recherche, publiés ou non, émanant des établissements d'enseignement et de recherche français ou étrangers, des laboratoires publics ou privés. 


\title{
ECG Fiducial Point Extraction Using Switching Kalman Filter
}

\author{
Mahsa Akhbaria,b,*, Nasim Montazeri Ghahjaverestan ${ }^{\mathrm{a}}$, Mohammad B. Shamsollahi ${ }^{\mathrm{a}}$, \\ Christian Jutten ${ }^{\mathrm{b}}$ \\ ${ }^{a}$ BiSIPL, Department of Electrical Engineering, Sharif university of Technology, Tehran, Iran. \\ ${ }^{b}$ GIPSA-Lab, Grenoble, and Institut Universitaire de France, France.
}

\begin{abstract}
In this paper, we propose a novel method for extracting fiducial points (FPs) of the beats in electrocardiogram (ECG) signals using switching Kalman filter (SKF). In this method, according to McSharry's model, ECG waveforms (P-wave, QRS complex and T-wave) are modeled with Gaussian functions and ECG baselines are modeled with first order auto regressive models. In the proposed method, a discrete state variable called "switch" is considered that affects only the observation equations. We denote a mode as a specific observation equation and switch changes between 7 modes and corresponds to different segments of an ECG beat. At each time instant, the probability of each mode is calculated and compared among two consecutive modes and a path is estimated, which shows the relation of each part of the ECG signal to the mode with the maximum probability. ECG FPs are found from the estimated path. For performance evaluation, the Physionet QT database is used and the proposed method is compared with methods based on wavelet transform, partially collapsed Gibbs sampler (PCGS) and extended Kalman filter. For our proposed method, the mean error and the root mean square error across all FPs are $2 \mathrm{~ms}$ (i.e. less than one sample) and $14 \mathrm{~ms}$, respectively. These errors are significantly smaller than those obtained using other methods. The proposed method achieves lesser RMSE and smaller variability with respect to others.
\end{abstract} Keywords: Electrocardiogram (ECG), Switching Kalman Filter (SKF), Extended

\footnotetext{
* Corresponding author

Email addresses: akhbari.mahsa@gmail.com (Mahsa Akhbari), montazeri.nasim@gmail.com (Nasim Montazeri Ghahjaverestan), mbshams@sharif .edu (Mohammad B. Shamsollahi), christian.jutten@gipsa-lab.grenoble-inp.fr (Christian Jutten)
} 
Kalman Filter (EKF), Segmentation, Fiducial Point (FP) Extraction.

\section{Introduction}

An electrocardiogram (ECG) describes the electrical activity of the heart. Onset, offset and peak location of ECG waves are known as fiducial points (FPs). Up to now, different methods have been used for detecting the QRS complex. See [1] for a review.

5 These methods are based on mathematical functions, filtering approaches (digital filters [2], adaptive filters [3]), classification methods (neural network approaches [4], support vector machine (SVM) [5], fuzzy C-means algorithm [6]), wavelet transform [7] and empirical mode decomposition (EMD) [8]. Low pass differentiation (LPD) [9], hidden Markov models (HMM) [10, 11, 12, 13, 14], partially collapsed Gibbs sampler (PCGS) [15], wavelet transform [16, 17, 18], correlation analysis [19, 20] and extended Kalman filter (EKF) [21, 22, 23, 24] are also used for ECG FP extraction.

FP extraction has been used as a preprocessing step in several applications such as detection of fragmented QRS complex [25], mobile health care applications [26], "Selvester QRS scoring" system [27], ischemia detection [28], ECG-based subject identification system[29] and biometric recognition based on fusion of ECG and EEG signals [30, 31].

Switching state space models are defined as the combination of HMMs and state space models [32]. When the model is linear and additive Gaussian noise exists, the switching state space models are known as "Switching Kalman Filter" (SKF) [33, 34]. In the SKF, at each time instance, the states are estimated by several Kalman filters (KFs). Furthermore, a hidden discrete state variable called switch is considered whose status changes over the time according to a Markov model. The switch indicates the KF which estimates the states better than others.

SKF is used for several applications such as figure tracking [35], acoustic segmentation [36], contour tracking in clutter [37], modeling and detecting motor cortical activity [38], prediction and tracking an adaptive meterological sensing network [39], tracking and event detection at traffic intersections [40], ECG ventricular beat classification [41] and finally for apnea bradycardia detection from ECG signals [42]. 
Although the methods based on dynamic models (EKF) [24] and sequential meth(which is combination of KF and HMM methods) have not been used for this application yet. The goal of this paper is showing the ability of SKF-based methods for ECG FP extraction.

In [21, 22, 23, 24], methods based on EKF have been proposed. The main limitation well as initial parameters of EKF, that must be defined by the user. Conversely, one of the advantages of the proposed SKF model is that it is not sensitive to the initial location of Gaussian functions and initial parameters of SKF.

According to McSharry's model [43], ECG waves (P-wave, QRS complex and T-

40 wave) are modeled with Gaussian functions. Baselines and segments between ECG waves are modeled with first order auto regressive (AR) models. In this SKF approach, a discrete switch affects only the observation equations and switches between 7 different values related to the 3 waves and the 4 baseline segments.

The performance of the proposed method is compared with previously published methods, including Wavelet [17], PCGS [15] and EKF-based method (EKF17) [22]. We also have a comparison with our previously proposed methods (linear and nonlinear EKF25 [23, 24]). Validation and comparison are done over Physionet QT database [44, 45].

The paper is organized as follows: ECG dynamical model and details of SKF approach for ECG FP extraction are described in Section 2 Section 3 presents the experimental results, and finally section 4 concludes the paper.

\section{Methods}

In this section, we first present the ECG model we used and then we fully describe the proposed SKF method.

\subsection{ECG Kalman Filtering Framework}

McSharry et al. [43] have proposed a synthetic ECG generator which is based on a nonlinear dynamic model. Sameni et al. [46] transformed it into polar coordinates 
from Cartesian coordinates and proposed an EKF-based framework which has two state variables and two corresponding observations. The discrete state-equations of this model are as follows:

$$
\left\{\begin{array}{l}
\varphi_{k+1}=\left(\varphi_{k}+\omega_{k} \delta\right) \bmod (2 \pi) \\
z_{k+1}=-\sum_{i} \delta \frac{\alpha_{i k} \omega_{k}}{b_{i k}^{2}} \Delta \theta_{i k} \exp \left(-\frac{\Delta \theta_{i k}^{2}}{2 b_{i k}^{2}}\right)+z_{k}+\eta_{k}
\end{array}\right.
$$

where $k$ denotes the discrete time, $\varphi_{k}$ is the phase of ECG and $\omega_{k}$ is the beat-to-beat angular frequency of the RR interval. In this model, $z_{k}$ is a state variable which is the sum of 5 Gaussian functions $(i \in\{P, Q, R, S, T\}$ ) and represents estimated amplitude of ECG. Each Gaussian function is defined with three parameters: $\alpha_{i k}, b_{i k}$ and $\theta_{i k}$, which correspond to the amplitude, width and location of the Gaussian functions and $\Delta \theta_{i k}=\left(\varphi_{k}-\theta_{i k}\right) \bmod (2 \pi) ; \delta$ is the sampling period and $\eta_{k}$ models the inaccuracies of the dynamic model.

\subsection{Proposed Dynamic Model}

For an ECG beat, we can define seven segments: $B_{1}, \mathrm{P}, \mathrm{PQ}\left(B_{2}\right), \mathrm{QRS}, \mathrm{ST}\left(B_{3}\right), \mathrm{T}$ and $B_{4}$ which are shown in Fig. 1 In the proposed model, we consider separate states

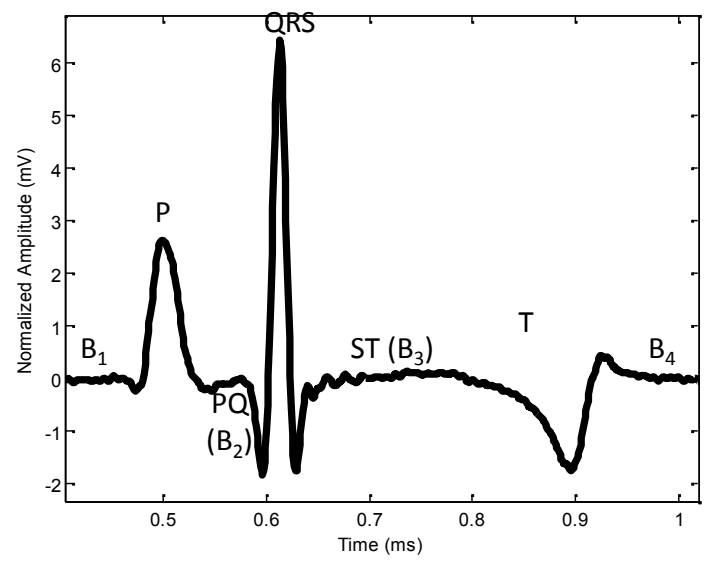

Figure 1: Segments of a single ECG beat.

for P-wave, QRS complex and T-wave which are modeled with Gaussian functions. We also assign a state defined with a first order AR model to each baseline $\left(B_{1}, B_{2}, B_{3}\right.$ 
and $B_{4}$ ). Similar to previous EKF-based models, we also consider the phase of ECG as a state. Hence, a model with 8 states is generated. In fact, although segments $B_{1}$ and $B_{4}$ are almost similar, since we find the fiducial points for each beat separately, we consider two separate segments $B_{1}$ and $B_{4}$. Discrete state and observation equations of this model are defined in (2) and (3), respectively. We use "C" to denote the QRS complex. In 2], for simplicity we consider that the coefficients of AR models are equal to one ( $\left.a_{B_{1}}=a_{B_{2}}=a_{B_{3}}=a_{B_{4}}=1\right)$ but in general, other values smaller and very close to 1 can be examined.

$$
\begin{aligned}
& \left\{\begin{array}{l}
\varphi_{k+1}=\left(\varphi_{k}+\omega_{k} \delta\right) \bmod (2 \pi) \\
B_{1, k+1}=a_{B_{1}} B_{1, k}+\eta_{B_{1, k}} \\
P_{k+1}=-\frac{\delta \alpha_{P_{P,}} \omega_{k}}{b_{P, k}} \Delta \theta_{P, k} \exp \left(-\frac{\Delta \theta_{P, k}^{2}}{2 b_{P, k}^{2}}\right)+P_{k}+\eta_{P_{k}} \\
B_{2, k+1}=a_{B_{2}} B_{2, k}+\eta_{B_{2, k}} \\
C_{k+1}=-\sum_{i \in\{Q, R, S\}} \delta \frac{\alpha_{i k} \omega_{k}}{b_{i k}^{2}} \Delta \theta_{i k} \exp \left(-\frac{\Delta \theta_{i k}^{2}}{2 b_{i k}^{2}}\right)+C_{k}+\eta_{C_{k}} \\
B_{3, k+1}=a_{B_{3}} B_{3, k}+\eta_{B_{3, k}} \\
T_{k+1}=-\frac{\delta \alpha_{T, k} \omega_{k}}{b_{T, k}^{2}} \Delta \theta_{T, k} \exp \left(-\frac{\Delta \theta_{T, k}^{2}}{2 b_{T, k}}\right)+T_{k}+\eta_{T_{k}} \\
B_{4, k+1}=a_{B_{4}} B_{4, k}+\eta_{B_{4, k}}
\end{array}\right. \\
& \left\{\begin{array}{l}
\Phi_{k}=\varphi_{k}+v_{1 k} \\
z_{k}=B_{1, k}+P_{k}+B_{2, k}+C_{k}+B_{3, k}+T_{k}+B_{4, k}+v_{2 k}
\end{array}\right.
\end{aligned}
$$

In (2), the first state is the phase of the ECG. States 3, 5 and 7 are distinct ECG waveforms. The ECG baselines are considered as the $2^{\text {nd }}, 4^{\text {th }}, 6^{\text {th }}$ and $8^{\text {th }}$ state variables. The system state and process noise vectors are defined as:

$$
\begin{aligned}
& \underline{x}_{k}=\left[\varphi_{k}, B_{1, k}, P_{k}, B_{2, k}, C_{k}, B_{3, k}, T_{k}, B_{4, k}\right]^{T} \\
& \underline{w}_{k}=\left[\alpha_{i_{k}}, b_{i_{k}}, \theta_{i_{k}}, \omega_{k}, \eta_{j_{k}}\right]^{T} \\
& i \in\{P, Q, R, S, T\}, j \in\left\{B_{1}, P, B_{2}, C, B_{3}, T, B_{4}\right\} .
\end{aligned}
$$

In (3), the first observation is a linearly approximated phase of ECG beat, and $z_{k}$ is the recorded ECG which can be considered as the sum of $B_{1, k}, P_{k}, B_{2, k}, C_{k}, B_{3, k}, T_{k}$ and $B_{4, k}$ states in the model. Observation and measurement noise vectors are defined respectively as: $\underline{y}_{k}=\left[\Phi_{k}, z_{k}\right]^{T}$ and $\underline{v}_{k}=\left[v_{1 k}, v_{2 k}\right]^{T}$. 


\subsection{SKF-based ECG model}

In our proposed SKF, we assume that the switch only affects the observation equation. We then denote a mode as a specific observation equation and the switch $s$ is a discrete state which changes between 7 modes, $j=1,2, \ldots, 7$. We assume to have a first order Markov chain whose matrix transition denotes as $Z$ where $z_{i j}=P\left(s_{k}=\right.$ $\left.j \mid s_{k-1}=i\right)$. The Markov chain has left-to-right structure i.e. $z_{i j} \neq 0$ if and only if $j=i$ or $j=i+1$ or $(j=7$ and $i=1)$. Since the model which is defined in $(2)$ is nonlinear, in order to use a Kalman filter for this system, it is necessary to derive a linear approximation of it near desired reference points $\left(\underline{\hat{x}}_{k}, \underline{\bar{w}}_{k}\right)$ and $\left(\underline{\hat{x}}_{k}^{-}, \underline{\underline{v}}_{k}\right)$. This approximation will lead to the following linear estimate:

$$
\left\{\begin{array}{l}
\underline{x}_{k+1}=f\left(\underline{x}_{k}, \underline{w}_{k}, k\right) \approx f\left(\underline{\hat{x}}_{k}, \overline{\bar{w}}_{k}, k\right)+A_{k}\left(\underline{x}_{k}-\underline{\hat{x}}_{k}\right)+D_{k}\left(\underline{w}_{k}-\underline{\bar{w}}_{k}\right) \\
\underline{y}_{k}=g\left(\underline{x}_{k}, \underline{v}_{k}, k\right) \approx g\left(\underline{\hat{x}}_{k}^{-}, \underline{\bar{v}}_{k}, k\right)+H_{k}\left(\underline{x}_{k}-\underline{\hat{x}}_{k}^{-}\right)+G_{k}\left(\underline{v}_{k}-\underline{\bar{v}}_{k}\right)
\end{array}\right.
$$

where

$$
\begin{aligned}
& A_{k}=\left.\frac{\partial f\left(\underline{\left.x_{k}, \underline{w}_{k}, k\right)}\right.}{\partial \underline{x}_{k}}\right|_{\underline{x}_{k}=\underline{\hat{x}}_{k}, \underline{w}_{k}=\underline{\bar{w}}_{k}}, \quad D_{k}=\left.\frac{\partial f\left(\underline{x}_{k}, \underline{w}_{k}, k\right)}{\partial \underline{w}_{k}}\right|_{\underline{x}_{k}=\underline{\hat{x}}_{k}, \underline{w}_{k}=\underline{\bar{w}}_{k}} \\
& H_{k}=\left.\frac{\partial g\left(\underline{x}_{k}, \underline{v}_{k}, k\right)}{\partial \underline{x}_{k}}\right|_{\underline{x}_{k}=\hat{x}_{k}^{-}, \underline{v}_{k}=\underline{\bar{v}}_{k}}, \quad G_{k}=\left.\frac{\partial g\left(\underline{x}_{k}, \underline{v}_{k}, k\right)}{\partial \underline{\underline{v}}_{k}}\right|_{\underline{x}_{k}=\hat{\hat{x}}_{k}^{-}, \underline{v}_{k}=\underline{\bar{v}}_{k}}
\end{aligned}
$$

The state and observation equations of the proposed SKF can be written as:

$$
\begin{aligned}
& \underline{x}_{k+1}=A_{k} \underline{x}_{k}+D_{k} \underline{w}_{k} \\
& \underline{y}_{k}=H_{k}^{(j)} \underline{x}_{k}+G_{k} \underline{v}_{k}^{(j)}
\end{aligned}
$$

where $H_{k}^{(j)}$ and $\underline{v}_{k}^{(j)}$ are the observation matrix and observation noise of the $j^{\text {th }}$ mode, respectively. Elements of $A_{k}$ and $D_{k}$ matrices are defined in (.1) and (.2), respectively in Appendix .1. Here, the matrices $A_{k}, D_{k}$ and $Q_{k}=\operatorname{Cov}\left(\underline{w}_{k}\right)$ are not changed for different modes. The dimension of $H_{k}^{(j)}$ is $2 \times 8$ and its nonzero elements are defined in $(8)$ :

$$
\left\{\begin{array}{l}
H_{k}^{(j)}(1,1)=1, j=1,2, \ldots, 7 \\
H_{k}^{(j)}(2, j+1)=1, j=1,2, \ldots, 7
\end{array}\right.
$$

This means that the observation equation has linear dynamics at each time instant but

70 it is time variant and switches among several linear equations over the time. $\underline{v}_{k}^{(j)}=$ $\left[v_{1 k}, v_{2 k}\right]^{T}$ is the observation noise vector. The noise covariance matrix $R_{k}^{(j)}=\operatorname{Cov}\left(\underline{v}_{k}^{(j)}\right)$ is defined from the observations of the $j^{\text {th }}$ mode. Matrix $G_{k}$ is constant for the different values of the switch: $G_{k}=I_{2}$ (identity matrix of size 2), $G_{k} \underline{v}_{k}^{(j)}=\underline{v}_{k}^{(j)}$ and $G_{k} R_{k}^{(j)} G_{k}^{T}=$ $R_{k}^{(j)}$. 


\subsection{Procedure of SKF-based FP extraction}

At each time instant $k$, the probability of each mode is calculated as: $K_{k}^{j}=P\left(s_{k}=\right.$ $\left.j \mid \underline{y}_{1: k}\right), \quad j \in\{1,2, \cdots, 7\}$. If $K_{k}^{j}$ has the maximum value at time $k$, the observation at time $k$ is likely generated by $j^{t h}$ mode. Monitoring the value of the parameter $K_{k}^{j}$, $j \in\{1,2, \cdots, 7\}$ for each mode allows us to choose the optimal mode. Therefore in this

80 work, we perform SKF and monitor $K_{k}^{j}$.

We use 2-fold cross validation [47] for each record (The records which are used in this paper will be explained in subsection 2.5. Initial values for $\underline{x}_{0}^{i}, P_{0}^{i}, Q_{0}, H_{0}^{(j)}, R_{0}^{(j)}$, $K_{0}^{i}$ and $Z_{0}(i, j=1,2, \cdots, 7)$ are found from train data in the Initialization step. $\underline{x}_{0}^{i}$ is the initial estimation of state and $P_{0}^{i}$ is its covariance matrix. $Q_{0}, H_{0}^{(j)}, R_{0}^{(j)}$ and $K_{0}^{i}$ are 85 initial values for $Q_{k}, H_{k}^{(j)}, R_{k}^{(j)}$ and $K_{k}^{i}$, respectively. Finally, $Z_{0}$ is the initial value of matrix $Z$ where $z_{i j}=P\left(s_{k}=j \mid s_{k-1}=i\right)$. In [48, 41], the estimation of the covariance matrix is done by performing a random search on the training data in order to optimize the covariance matrix parameters. In this work, for defining the initial location of the Gaussians, we follow the same procedure of Sameni et al. [46]. we choose manually the initial location of the Gaussians and then doing a curve fitting. For estimation of the covariance matrices, we follow the same procedure as [46].

Here, since the matrices $A_{k}, D_{k}$ and $Q_{k}$ are not changed for different modes, the training step includes only the initialization step and no parameter is trained in this step. After that we perform inference procedure (in one iteration) as shown in Algorithm 1 , below. Functions filter, StatesProbability and Collapse and their parameters are defined in Appendix .2.

In the function filter, $\underline{x}_{k}^{-i j}=E\left\{\underline{x}_{k} \mid \underline{y}_{1: k-1}, s_{k}=j, s_{k-1}=i\right\}$ is the prior estimate of the state vector, in the $k^{\text {th }}$ stage, using the past observations $\underline{y}_{0}$ to $\underline{y}_{k-1}$ and $\underline{x}_{k}^{i j}=$ $E\left\{\underline{x}_{k} \mid \underline{y}_{1: k}, s_{k}=j, s_{k-1}=i\right\}$ is the posterior estimate of this state vector after adding the $100 k^{\text {th }}$ observations $\underline{y}_{k} . P_{k}^{-i j}$ and $P_{k}^{i j}$ are defined in the same manner to be the prior and posterior estimates of the covariance matrices, in the $k^{\text {th }}$ stage, before and after using the $k^{\text {th }}$ observation, respectively. $L_{k}^{i j}=p\left(\underline{y}_{k} \mid \underline{y}_{1: k-1}, s_{k}=j, s_{k-1}=i\right)$ is the likelihood of observing the $k^{\text {th }}$ observation $\underline{y}_{k}$.

The function StatesProbability, computes the probabilistic parameters $K_{k}^{j}=P\left(s_{k}=\right.$ $\left.105 j \mid \underline{y}_{1: k}\right), g_{k}^{i j}=P\left(s_{k-1}=i \mid \underline{y}_{1: k}, s_{k}=j\right)$ and $K_{k}^{i j}=P\left(s_{k-1}=i, s_{k}=j \mid \underline{y}_{1: k}\right)=K_{k}^{j} g_{k}^{i j}$. 
Finally, the function Collapse approximates mixture of Gaussians as a unique Gaussian by moment matching. The mean vector and covariance matrix of the unique Gaussian are the same as that of the mixture and are computed as: $\underline{x}_{k}^{j}=E\left\{\underline{x}_{k} \mid \underline{y}_{1: k}, s_{k}=j\right\}$ and $P_{k}^{j}=\operatorname{Cov}\left\{\underline{x}_{k} \mid \underline{y}_{1: k}, s_{k}=j\right\}$ from mixture model using an approximation of $p\left(\underline{x}_{k} \mid \underline{y}_{1: k}, s_{k}=\right.$ $j)=\mathscr{N}\left(\underline{x}_{k}^{j}, P_{k}^{j}\right)$. More information about SKF functions can be found in [38, 42].

Algorithm 1: SKF: Inference

Inputs: $x_{0}^{i}, P_{0}^{i}, Q_{0}, H_{0}^{(j)}, R_{0}^{(j)}, K_{0}^{i}$ and $Z_{0}, i, j=1,2, \cdots, 7$.

Outputs: $K_{k}^{j}$

1: for $k=1,2, \cdots, T$ do

2: $\quad$ Compute $A_{k}$ and $D_{k}$ from $(.1)$ and $(.2)$ in Appendix .1.

3: $\quad$ for $i, j=1,2, \cdots, 7$ do

4: $\quad\left[\underline{x}_{k}^{i j}, P_{k}^{i j}, L_{k}^{i j}\right]=\operatorname{filter}\left(\underline{x}_{k-1}^{i}, P_{k-1}^{i}, A_{k}, D_{k}, Q_{k}, H_{k}^{(j)}, R_{k}^{(j)}\right)$

5: $\quad\left[K_{k}^{i j}, K_{k}^{j}, g_{k}^{i j}\right]=$ StatesProbability $\left(L_{k}^{i j}, z_{i j}, K_{k-1}^{i}\right)$

6: $\quad\left[\underline{x}_{k}^{j}, P_{k}^{j}\right]=$ Collapse $\left(\underline{x}_{k}^{i j}, P_{k}^{i j}, K_{k}^{j}, g_{k}^{i j}\right)$

7: $\quad$ end for

8: end for

9: Applying a threshold on $K_{k}^{j}$

The procedure of finding $K_{k}^{j}$ is done for each ECG beat, separately. Since each ECG beat starts with $B_{1}$ segment, we assume that $K_{0}^{1}=1$ and $K_{0}^{i}=0, i=2, \cdots, 7$.

For each time instant $k, K_{k}^{j}$ has seven values according to the values which the switch can obtain. Since switch $s$ has a left-to-right HMM model, we find the optimum mode between two consecutive modes by:

$$
\text { mode }_{\text {opt }}=\underset{j}{\operatorname{argmax}} K_{k}^{j}, \quad j=\{k k, k k+1\}, k k \in\{1,2, \cdots, 6\}
$$

Where $k k$ is the current state. After finding the "mode $e_{\text {opt }}$ " values, a path is estimated from these values. The estimated path has seven levels, each one associated to one segment. Levels 1 to 7 represent the $B_{1}, \mathrm{P}, B_{2}$, QRS, $B_{3}$, T and $B_{4}$ segments, respectively (like Fig 3). The proposal to find the onset and offset of waves from the estimated path is as follows: 
- $P_{o n}$ : The point in which the path transits from level 1 to 2

- $P_{o f f}$ : The point in which the path transits from level 2 to 3

- $Q R S_{\text {off }}$ : The point in which the path transits from level 4 to 5

- $T_{o n}$ : The point in which the path transits from level 5 to 6

- $T_{o f f}$ : The point in which the path transits from level 6 to 7

Since the peaks can be positive or negative, peak position of waves $\left(P_{\text {peak }}, R_{\text {peak }}, T_{\text {peak }}\right)$ are defined as the maximum of absolute value of signal between onset and offset.

\subsection{Data and Evaluation Metrics}

To evaluate the performance of the proposed method in extracting ECG fiducial points, we need ECG recordings annotated by physicians. Thus, we use records of Arrhythmia, Normal Sinus Rhythm, ST Change and Supraventricular databases which are annotated in the Physionet QT database (32 records) [44, 45]. The records are sampled at $250 \mathrm{~Hz}$ (4 ms between 2 successive samples) and each of them has 30-50 annotated beats. Totally we use 975 annotated beats for evaluation of the performance of the methods.

We use 2-fold cross validation for each record and the initial parameters of SKF model are found from train data.

For quantitative evaluation of a FP extraction method, we calculate the estimation error defined as time differences between estimated points by proposed method and cardiologist annotations (considered as ground truth). Quantitative results are reported using common metrics: mean (m), standard deviation (sd) and root mean square error (RMSE), defined as follows:

$$
R M S E=\sqrt{M S E}=\sqrt{\frac{1}{N} \sum_{i=1}^{N}\left(e_{i}\right)^{2}}=\sqrt{\left(m^{2}+s d^{2}\right)}
$$

where $e_{i}=\hat{y}_{i}-y_{i}$ is denoted as the $i^{\text {th }}$ element of the estimation error vector and $N$ is the length of error vector. $y_{i}$ and $\hat{y}_{i}$ are the $i^{\text {th }}$ cardiologist annotation and estimated 
point, respectively. $m, s d$ and $R M S E$ are given in ms. Since RMSE considers mean and standard deviation of error, it is a more relevant parameter for comparing methods.

\section{Results}

Fig 2 shows how the estimated path is obtained from comparing the values of $K_{k}^{j}$ parameter. In this figure, the upper subfigure shows the calculated $K_{k}^{j}, j=1, \cdots, 7$ probabilities for one beat. We can see that for example $K_{k}^{1}$ has high value on the segment which is related to $B_{1}$ segment and has low (almost zero) value in other segments and so on. By comparing the two consecutive $K_{k}^{j}$ values by 9 , we can obtain the mode $_{\text {opt }}$ and the estimated path which is plotted in lower subfigure in Fig. 2 .
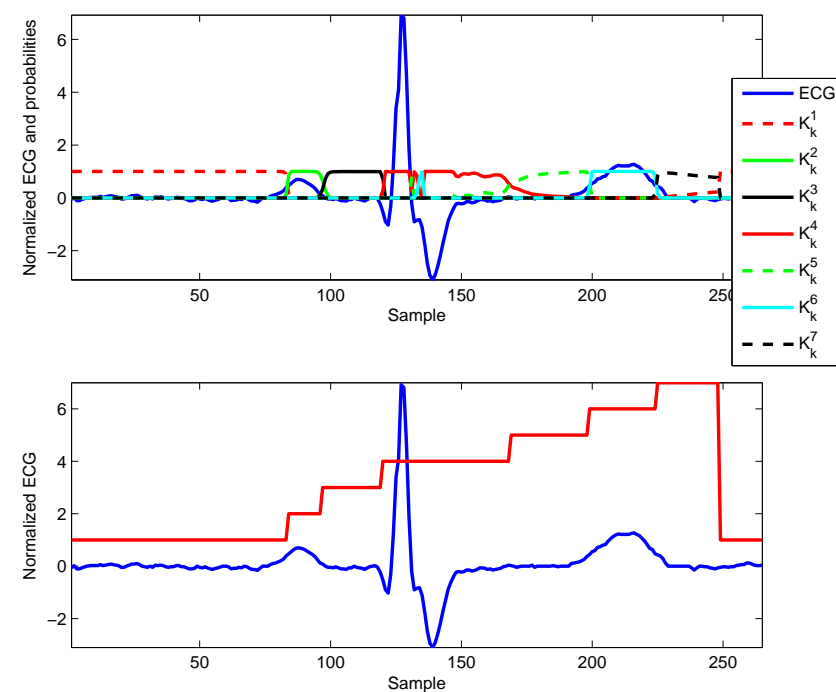

Figure 2: The $k_{k}^{j}$ values and estimated path by SKF for record Sel231.

Fig 3 shows the estimated path by the SKF for a small segment of the record 231 of the QT database. The colorful vertical lines show the original location of FPs which are annotated by a physician and the multilevel path is estimated by the proposed SKF. This figure is an illustrative example of what the estimated path looks like and clarify how the onset and offset of waves can be found from the transition of one level to upper level in a multi-level estimated path. 


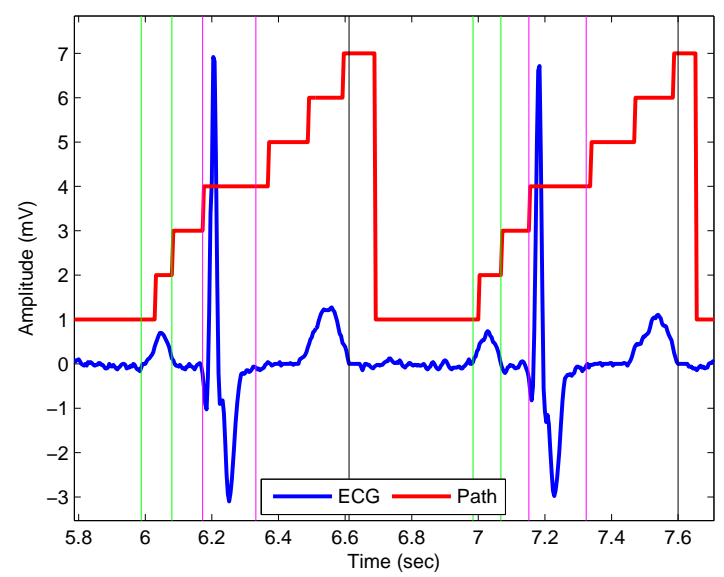

Figure 3: Original labels and estimated path by SKF for record Sel231.

Here, we first present the quantitative results of proposed SKF for some records with different morphologies. Afterthat, we compare the estimation error of SKF and 

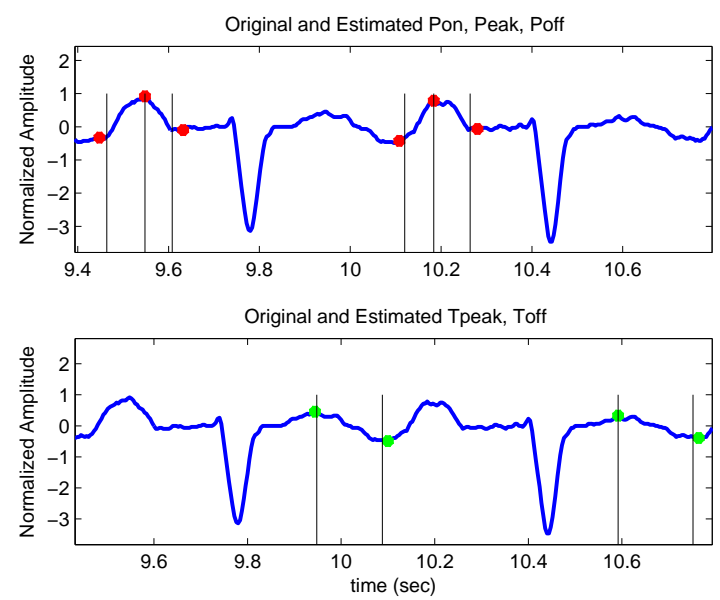

Figure 4: Original and estimated onset, peak and offset of P- and T-waves by SKF for record Sel41.

The quantitative results of different methods for ECG FP extraction for 32 records of QT database are compared in Tables 1 and 2 In Table 1 , we compare the proposed SKF method with other methods of the literature: Wavelet[17], PCGS[15] and EKFbased method (EKF17)[22]. In Table 2, we compare SKF with our previously proposed methods based on EKF: linear and nonlinear EKF25 methods proposed in [23] and [24]. Most of the records of QT database do not have reference label for $T_{o n}$. Our proposed method can estimate the location of $T_{\text {on }}$ but we can not report the results of estimation error for $T_{o n}$ due to lack of reference labels.

Table 1: Mean \pm standard deviation (first line) and RMSE (second line) of error in ms between estimated FPs and manual annotations for signals of the QT database (fs $=250 \mathrm{~Hz})$, (N.A.: Not Available)

\begin{tabular}{|l|c|c|c|c|c|c|c|c|}
\hline Method & $P_{\text {on }}$ & $P_{\text {peak }}$ & $P_{\text {off }}$ & $Q R S_{\text {on }}$ & $R_{\text {peak }}$ & $Q R S_{\text {off }}$ & $T_{\text {peak }}$ & $T_{\text {off }}$ \\
\hline SKF & $23.4 \pm 15.2$ & $-0.1 \pm 1.5$ & $-6.4 \pm 20$ & $6.6 \pm 10.2$ & $0.01 \pm 0.1$ & $-5.7 \pm 8.5$ & $-0.01 \pm 0.4$ & $0.6 \pm 10.8$ \\
& $\mathbf{2 7 . 8}$ & $\mathbf{1 . 5}$ & 21 & $\mathbf{1 2}$ & $\mathbf{0 . 1}$ & $\mathbf{1 0 . 3}$ & $\mathbf{0 . 4}$ & $\mathbf{1 0 . 8}$ \\
\hline Wavelet [17] & $-2.3 \pm 31.6$ & $0.7 \pm 25$ & $2.6 \pm 15.2$ & $12.4 \pm 13.6$ & $1.4 \pm 3.6$ & $1.9 \pm 13.8$ & $7.5 \pm 27.5$ & $7.3 \pm 32.2$ \\
& 31.7 & 25 & $\mathbf{1 5 . 4}$ & 18.4 & 3.8 & 13.9 & 28.5 & 33 \\
\hline PCGS [15] & $-30 \pm 29.4$ & $5.2 \pm 8.3$ & $21.1 \pm 15.5$ & N.A & N.A & N.A & $2.6 \pm 29.7$ & $27.5 \pm 44$ \\
& 42 & 9.8 & 26.2 & N.A & N.A & N.A & 29.8 & 51.8 \\
\hline EKF17 [22] & $-11 \pm 28.7$ & $9 \pm 19.2$ & $27.4 \pm 23.5$ & $-24.5 \pm 39.2$ & $4.5 \pm 6.4$ & $22.2 \pm 40.6$ & $-4.6 \pm 34$ & $24.8 \pm 44.5$ \\
& 30.7 & 21.2 & 36.1 & 46.2 & 7.8 & 46.2 & 34.3 & 51 \\
\hline
\end{tabular}



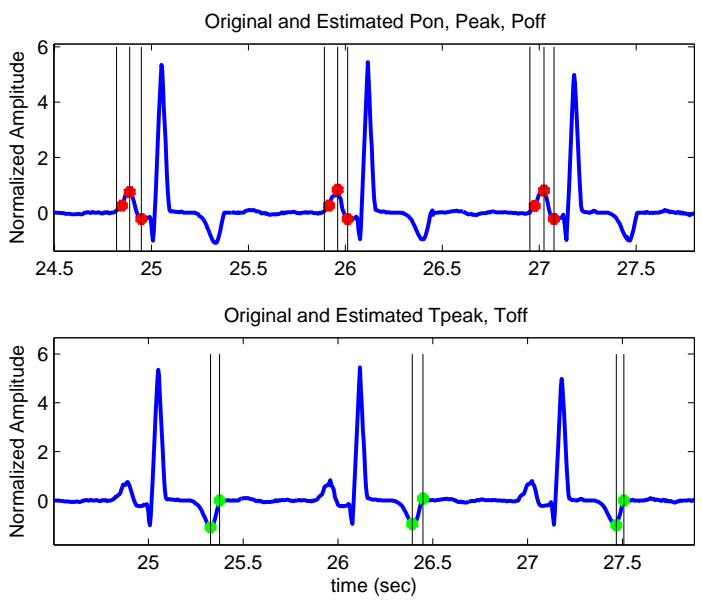

Figure 5: Original and estimated onset, peak and offset of P- and T-waves by SKF for record Sel808. ods (linear and nonlinear EKF25 methods) in Table 2. The results show that for all FPs except $P_{o n}, P_{o f f}$ and $Q R S_{\text {off }}$, SKF has better results that EKF25 methods. 


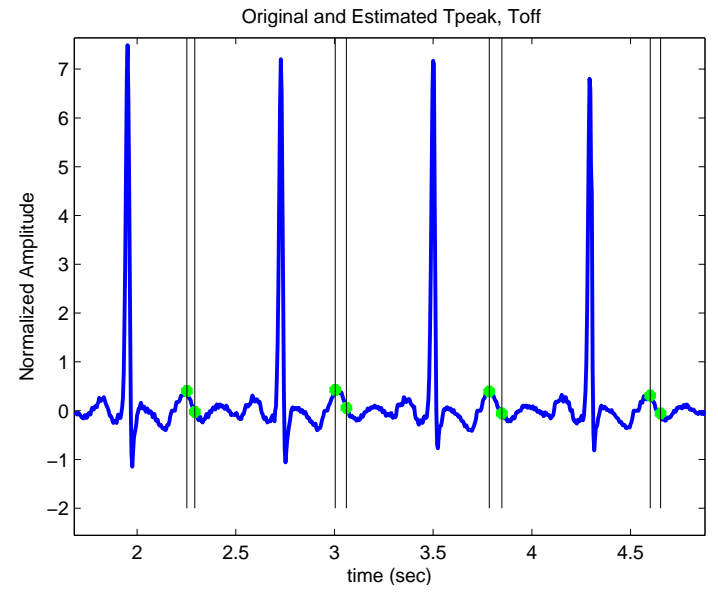

Figure 6: Original and estimated peak and offset of T-waves by SKF for record Sel301.

The simulations were done using a system with Core i3, $2.53 \mathrm{GHz}$ CPU. The runtime of the SKF method for one second record takes about 3 seconds.

Table 2: Mean \pm standard deviation (first line) and RMSE (second line) of error in ms between estimated FPs and manual annotations for signals of the QT database ( $\mathrm{fs}=250 \mathrm{~Hz}$ ), Methods: SKF, Linear EKF25 (L.EKF25) and Nonlinear EKF25 (N.EKF25)

\begin{tabular}{|l|c|c|c|c|c|c|c|c|}
\hline Method & $P_{\text {on }}$ & $P_{\text {peak }}$ & $P_{\text {off }}$ & $Q R S_{\text {on }}$ & $R_{\text {peak }}$ & $Q R S_{\text {off }}$ & $T_{\text {peak }}$ & $T_{\text {off }}$ \\
\hline SKF & $23.4 \pm 15.2$ & $-0.1 \pm 1.5$ & $-6.4 \pm 20$ & $6.6 \pm 10.2$ & $0.01 \pm 0.1$ & $-5.7 \pm 8.5$ & $-0.01 \pm 0.4$ & $0.6 \pm 10.8$ \\
& 27.8 & $\mathbf{1 . 5}$ & 21 & $\mathbf{1 2}$ & $\mathbf{0 . 1}$ & 10.3 & $\mathbf{0 . 4}$ & $\mathbf{1 0 . 8}$ \\
\hline L. EKF25 [23] & $0.9 \pm 14.7$ & $4 \pm 10.9$ & $-0.1 \pm 11.7$ & $0.55 \pm 12.3$ & $1.5 \pm 2.7$ & $-2.5 \pm 7$ & $-0.6 \pm 9.7$ & $0.3 \pm 14.7$ \\
& $\mathbf{1 4 . 8}$ & 11.6 & $\mathbf{1 1 . 7}$ & 12.3 & 3.1 & 7.3 & 9.7 & 14.7 \\
\hline N. EKF25 [24 & $-1.9 \pm 26.7$ & $3.4 \pm 15$ & $-1.6 \pm 13.4$ & $1 \pm 12.7$ & $1.2 \pm 5.3$ & $-1.7 \pm 7$ & $-1 \pm 8.8$ & $-0.4 \pm 11.5$ \\
& 26.8 & 15.4 & 13.5 & 12.7 & 5.5 & $\mathbf{7 . 2}$ & 8.8 & 11.5 \\
\hline
\end{tabular}

\section{Discussion and Conclusions}

In this paper, a novel method based on SKF for ECG fiducial point extraction is proposed. Experiments carried out on ECG signals from the QT database show that the SKF performance is similar or better than the state of the art ECG delineators such as EKF17, PCGS and Wavelet. For most of FPs, SKF outperforms linear and nonlinear 


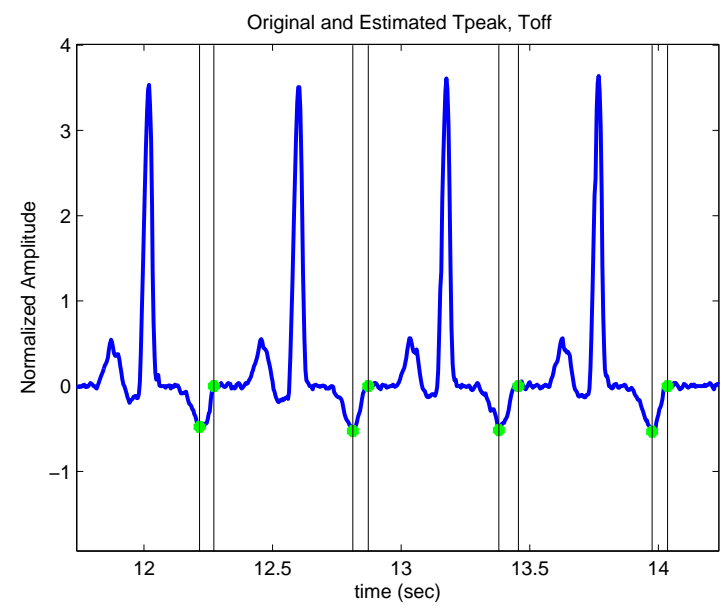

Figure 7: Original and estimated peak and offset of T-waves by SKF for record Sel233.

EKF25 methods.

The main contribution of this paper is proposing a SKF model for ECG FP extraction, which for each ECG wave or segment, a separate state is considered. ECG waves are modeled as a sum of 5 Gaussian functions and ECG baselines are modeled with a first order AR model. The switch only affects the observations. We used 2-fold cross validation and the initial parameters are found from train data. In the test step, the probability of $K_{k}^{j}=P\left(s_{k}=j \mid y_{1: k}\right)$ is compared among all modes and a path is estimated, which shows the relation of each part of the ECG signal to the mode with the maximum probability. FPs are directly found from the obtained path.

EKF-based approaches have been previously used for ECG FP extraction [21, 22, 23, 24]. The main limitation of such methods is their sensitivity to the initial location of the Gaussian functions as well as initial parameters of EKF, that must be defined by the user. HMM-based approaches have also been previously proposed for ECG FP extraction [10, 11, 12, 13]. In these methods, defining a suitable HMM structure, training step and finding the HMM parameters are very critical and methods are sensitive to these points.

The advantages of the proposed SKF model are: (i) It is sensitive neither to the initial location of the Gaussian functions nor to the initial parameters of SKF, (ii) It 
does not require severe training step.

The main goal of this paper, is to show the ability of SKF-based methods for ECG

FP extraction. The complexity analysis and adapting the algorithm for real-time application are not in the scope of the paper and can be considered as future works.

\section{Acknowledgment}

This work has been partly supported by the PhD scholarship of the French Embassy.

\section{References}

[1] B. Kohler, C. Hennig, R. Orglmeister, The principles of software QRS detection: Reviewing and comparing algorithms for detecting this important ECG waveform, IEEE Engineering in Medicine and Biology (2002) 42-57.

[2] B. C. Yu, S. Liu, M. Lee, C. Y. Chen, B. N. Chiang, A nonlinear digital filter for cardiac QRS complex detection, Journal of Clinical Engineering 10 (1985) 193-201.

[3] E. Soria, M. Sober, J. Calpe, J. Francisco, J. Chorro, J. Lopez, Application of adaptive signal processing for determining the limits of $\mathrm{P}$ and $\mathrm{T}$ waves in an ECG, IEEE Transaction on Biomedical Engineering 45 (8) (1998) 1077-1080.

[4] Y. H. Hu, W. J. Tompkins, J. L. Urrusti, V. X. Afonso, Applications of artificial neural networks for ECG signal detection and classification, Journal of Electrocardiology 26 (1993) 66-73.

[5] S. S. Mehta, N. S. Lingayat, Combined entropy based method for detection of QRS complexes in 12-lead electrocardiogram using SVM, Computers in Biology and Medicine 38 (2008) 138-145.

[6] S. S. Mehta, C. R. Trivedi, N. S. Lingayat, Identification and delineation of QRS complexes in electrocardiogram using fuzzy C-means algorithm, Journal of Theoretical and Applied Information Technology (2009) 609-617. 
[7] M. Merah, T. A. Abdelmalik, B. H. Larbi, R-peaks detection based on stationary wavelet transform, Computer Methods and Programs in Biomedicine 121 (2015) $149-160$.

[8] S. Pal, M. Mitra, Empirical mode decomposition based ECG enhancement and QRS detection, Computers in Biology and Medicine 42 (2012) 83-92.

[9] P. Laguna, R. Jane, R. Caminal, Automatic detection of wave boundaries in multilead ECG signals: validation with the CSE data-base, Computers and Biomedical Research 27 (1994) 45-60.

[10] D. A. Coast, R. M. Stern, G. G. Cano, S. A. Briller, An approach to cardiac arrhythmia analysis using hidden Markov models, IEEE Transaction on Biomedical Engineering. 37 (9) (1990) 826-836.

[11] N. P. Hughes, Probabilistic models for automated ECG interval analysis, Ph.D. thesis, Department of Engineering Science, University of Oxford (2006).

[12] R. Andreao, B. Dorizzi, J. Boudy, ECG signal analysis through hidden Markov models, IEEE Transaction on Biomedical Engineering 53 (8) (2006) 1541-1549.

[13] R. Andreao, J. Boudy, Combining wavelet transform and hidden Markov models for ECG segmentation, EURASIP Journal on Advances in Signal Processing (2006) 1-6.

[14] M. Akhbari, M. B. Shamsollahi, O. Sayadi, A. A. Armoundas, C. Jutten, ECG segmentation and fiducial point extraction using multi hidden Markov model, Computers in Biology and Medicine 79 (2016) 21-29.

[15] C. Lin, C. Mailhes, J. Y. Tourneret, P- and T-wave delineation in ECG signals using a Bayesian approach and a partially collapsed Gibbs sampler, IEEE Transaction on Biomedical Engineering 57 (12) (2010) 2840-2849.

[16] C. Li, C. Zheng, C. Tai, Detection of ECG characteristic points using wavelet transforms, IEEE Transaction on Biomedical Engineering 42 (1) (1995) 21-28. 
[17] J. P. Martinez, R. Almeida, S. Olmos, A. P. Rocha, P. Laguna, A wavelet-based ECG delineator: Evaluation on standard databases, IEEE Transaction on Biomedical Engineering 51 (4) (2004) 570-581.

[18] J. Dumont, A. I. Hernandez, G. Carrault, Improving ECG beats delineation with an evolutionary optimization process, IEEE Transaction on Biomedical Engineering 57 (2010) 607-615.

[19] M. R. Homaeinezhad, M. E. Moshiri-Nejad, H. Naseri, A correlation analysisbased detection and delineation of ECG characteristic events using template waveforms extracted by ensemble averaging of clustered heart cycles, Computers in Biology and Medicine 44 (2014) 66-75.

[20] A. Karimipour, M. R. Homaeinezhad, Real-time electrocardiogram P-QRS-T detection-delineation algorithm based on quality-supported analysis of characteristic templates, Computers in Biology and Medicine 52 (2014) 153-165.

[21] M. Akhbari, M. B. Shamsollahi, C. Jutten, Fiducial points extraction and characteristic waves detection in ECG signal using a model-based Bayesian framework, in: International Conference on Acoustics, Speech, and Signal Processing, 2013, pp. 1257-1261.

[22] O. Sayadi, M. B. Shamsollahi, A model-based Bayesian framework for ECG beat segmentation, Physiological Measurement 30 (2009) 335-352.

[23] M. Akhbari, M. B. Shamsollahi, C. Jutten, ECG fiducial points extraction by extended Kalman filtering, in: International Conference on Telecommunications and Signal Processing, 2013, pp. 628-632.

[24] M. Akhbari, M. B. Shamsollahi, C. Jutten, A. A. Armoundas, O. Sayadi, ECG denoising and fiducial point extraction using extended Kalman filtering framework with linear and nonlinear phase observation, Physiological Measurement Journal 37 (2016) 203-226.

[25] S. Maheshwari, A. Acharyya, P. E. Puddu, E. B. Mazomenos, G. Leekha, K. Maharatna, M. Schiariti, An automated algorithm for online detection of fragmented 
QRS and identification of its various morphologies, Journal of the Royal Society 10 (2013) 1-18.

[26] E. B. Mazomenos, D. Biswas, A. Acharyya, T. Chen, K. Maharatna, J. Rosen-

[31] S. Barra, A. Casanova, M. Fraschini, M. Nappi, Fusion of physiological measures for multimodal biometric systems, Multimedia Tools and Applications (2016) 113.

[32] Z. Ghahramani, G. E. Hinton, Variational learning for switching state-space mod325

[33] K. P. Murphy, Switching Kalman filters, Tech. rep., Citeseer (1998).

[34] Z. Ghahramani, G. E. Hinton, Switching state space models, Tech. rep. (1998). 
[35] V. Pavlovic, J. M. Rehg, T. Cham, K. P. Murphy, A dynamic Bayesian network approach to figure tracking using learned dynamic models, in: IEEE International Conference on Computer Vision, 1999, pp. 94-101.

[36] Y. Zheng, M. Hasegawa-Johnson, Acoustic segmentation using switching state Kalman filter, in: IEEE International Conference on Acoustics, Speech, and Signal Processing, 2003, pp. 752-755.

[37] Y. Wu, G. Hua, T. Yu, Switching observation models for contour tracking in clutter, in: IEEE Conference on Computer Vision and Pattern Recognition, 2003.

[38] W. Wu, M. J. Black, D. Mumford, Y. Gao, E. Bienenstock, J. P. Donoghue, Modeling and decoding motor cortical activity using a switching Kalman filter, IEEE Transaction on Biomedical Engineering 51 (6) (2004) 933-942.

[39] V. Manfredi, S. Mahadevan, J. F. Kurose, Switching Kalman filters for prediction and tracking in an adaptive meteorological sensing network, in: IEEE Conference on Sensor and Ad Hoc Communications and Networks, 2005, pp. 197-206.

[40] H. Veeraraghavan, P. Schrater, N. Papanikolopoulos, Switching Kalman filterbased approach for tracking and event detection at traffic intersections, in: Mediterranean Conference on Control and Automation, 2005, pp. 1167-1172.

[41] J. Oster, J. Behar, O. Sayadi, S. Nemati, A. E. W. Johnson, G. D. Clifford, Semi supervised ECG ventricular beat classification with novelty detection based on switching Kalman filters, IEEE Transaction on Biomedical Engineering 62 (9) (2015) 2125-2134.

[42] N. Montazeri, M. B. Shamsollahi, D. Ge, A. I. Hernandez, Switching Kalman filter based methods for apnea bradycardia detection from ECG signals, Physiological Measurement 36 (9) (2015) 1763-1783.

[43] P. E. McSharry, G. D. Clifford, L. Tarassenko, L. A. Smith, A dynamic model for generating synthetic electrocardiogram signals, IEEE Transaction on Biomedical Engineering 50 (3) (2003) 289-294. 
[44] http://www.physionet.org/physiobank/database/qtdb.

[45] P. Laguna, R. G. Mark, A. Goldberg, G. B. Moody, A database for evaluation of algorithms for measurement of QT and other waveform intervals in the ECG, IEEE, Computers in Cardiology 24 (1997) 673-676.

[46] R. Sameni, M. B. Shamsollahi, C. Jutten, G. D. Clifford, Nonlinear Bayesian filtering framework for ECG denoising, IEEE Transaction on Biomedical Engineering 54 (12) (2007) 2172-2185.

[47] R. Kohavi, A study of cross validation and bootstrap for accuracy estimation and model selection, in: International Joint Conference on Artificial Intelligence, 1995.

[48] J. Behar, J. Oster, G. D. Clifford, Combining and benchmarking methods of foetal ECG extraction without maternal or scalp electrode data, Physiological Measurement 35 (2014) 1569-1589.

\section{Appendix.1. EKF matrices derivations}

In order to set up an EKF model based on the nonlinear synthetic model of (2), it is necessary to have a linearized version of the model. Consequently, the state-equation of (2) requires linearization using (5) and (6). The following equations represent the linearized model with respect to the state variables (non-zero elements of matrix $A_{k}$ ):

$$
\begin{aligned}
& A_{k}(1,1)=A_{k}(2,2)=A_{k}(3,3)=A_{k}(4,4)=A_{k}(5,5)=A_{k}(6,6)=A_{k}(7,7)=1 \\
& A_{k}(3,1)=-\delta \frac{\alpha_{P, k} \omega_{k}}{b_{P, k}^{2}}\left[1-\frac{\Delta \theta_{P, k}^{2}}{b_{P, k}^{2}}\right] \exp \left(-\frac{\Delta \theta_{P, k}^{2}}{2 b_{P, k}^{2}}\right) \\
& A_{k}(5,1)=-\sum_{i \in\{Q, R, S\}} \delta \frac{\alpha_{i k} \omega_{k}}{b_{i k}^{2}}\left[1-\frac{\Delta \theta_{i k}^{2}}{b_{i k}^{2}}\right] \exp \left(-\frac{\Delta \theta_{i k}^{2}}{2 b_{i k}^{2}}\right) \\
& A_{k}(7,1)=-\delta \frac{\alpha_{T, k} \omega_{k}}{b_{T, k}^{2}}\left[1-\frac{\Delta \theta_{T, k}^{2}}{b_{T, k}^{2}}\right] \exp \left(-\frac{\Delta \theta_{T, k}^{2}}{2 b_{T, k}^{2}}\right)
\end{aligned}
$$


Similarly, the linearization of (2) with respect to the process noise components yields

$$
\begin{aligned}
& D_{k}(1,16)=\delta, D_{k}(2,17)=D_{k}(4,19)=D_{k}(6,21)=D_{k}(8,23)=1 \\
& D_{k}(3,18)=D_{k}(5,20)=D_{k}(7,22)=1 \\
& D_{k}(3,1)=-\delta \frac{\omega_{k} \Delta \theta_{P, k}}{b_{P, k}^{2}} \exp \left(\frac{-\Delta \theta_{P, k}^{2}}{2 b_{P, k}^{2}}\right) \\
& D_{k}(3,6)=2 \delta \frac{\alpha_{P, k} \omega_{k} \Delta \theta_{P, k}}{b_{P, k}^{3}}\left(1-\frac{\Delta \theta_{P, k}^{2}}{2 b_{P, k}^{2}}\right) \exp \left(\frac{-\Delta \theta_{P, k}^{2}}{2 b_{P, k}^{2}}\right) \\
& D_{k}(3,11)=\delta \frac{\alpha_{P, k} \omega_{k}}{b_{P, k}^{2}}\left(1-\frac{\Delta \theta_{P, k}^{2}}{b_{P, k}^{2}}\right) \exp \left(\frac{-\Delta \theta_{P, k}^{2}}{2 b_{P, k}^{2}}\right) \\
& D_{k}(3,16)=-\delta \frac{\alpha_{P, k} \Delta \theta_{P, k}}{b_{P, k}^{2}} \exp \left(-\frac{\Delta \theta_{P, k}^{2}}{2 b_{P, k}^{2}}\right) \\
& D_{k}(5,2: 4)=-\delta \frac{\omega_{k} \Delta \theta_{i k}}{b_{i k}^{2}} \exp \left(\frac{-\Delta \theta_{i k}^{2}}{2 b_{i k}^{2}}\right), i \in\{Q, R, S\} \\
& D_{k}(5,7: 9)=2 \delta \frac{\alpha_{i k} \omega_{k} \Delta \theta_{i k}}{b_{i k}^{3}}\left(1-\frac{\Delta \theta_{i k}^{2}}{2 b_{i k}^{2}}\right) \exp \left(\frac{-\Delta \theta_{i k}^{2}}{2 b_{i k}^{2}}\right), i \in\{Q, R, S\} \\
& D_{k}(5,12: 14)=\delta \frac{\alpha_{i k} \omega_{k}}{b_{i k}^{2}}\left(1-\frac{\Delta \theta_{i k}^{2}}{b_{i k}^{2}}\right) \exp \left(\frac{-\Delta \theta_{i k}^{2}}{2 b_{i k}^{2}}\right), i \in\{Q, R, S\} \\
& D_{k}(5,16)=-\sum_{i \in\{Q, R, S\}} \delta \frac{\alpha_{i k} \Delta \theta_{i k}}{b_{i k}^{2}} \exp \left(-\frac{\Delta \theta_{i k}^{2}}{2 b_{i k}^{2}}\right) \\
& D_{k}(7,5)=-\delta \frac{\omega_{k} \Delta \theta_{T, k}}{b_{T, k}^{2}} \exp \left(\frac{-\Delta \theta_{T, k}^{2}}{2 b_{T, k}^{2}}\right) \\
& D_{k}(7,10)=2 \delta \frac{\alpha_{T, k} \omega_{k} \Delta \theta_{T, k}}{b_{T, k}^{3}}\left(1-\frac{\Delta \theta_{T, k}^{2}}{2 b_{T, k}^{2}}\right) \exp \left(\frac{-\Delta \theta_{T, k}^{2}}{2 b_{T, k}^{2}}\right) \\
& D_{k}(7,15)=\delta \frac{\alpha_{T, k} \omega_{k}}{b_{T, k}^{2}}\left(1-\frac{\Delta \theta_{T, k}^{2}}{b_{T, k}^{2}}\right) \exp \left(\frac{-\Delta \theta_{T, k}^{2}}{2 b_{T, k}^{2}}\right) \\
& D_{k}(7,16)=-\delta \frac{\alpha_{T, k} \Delta \theta_{T, k}}{b_{T, k}^{2}} \exp \left(-\frac{\Delta \theta_{T, k}^{2}}{2 b_{T, k}^{2}}\right)
\end{aligned}
$$


Appendix .2. Functions of Switching Kalman Filter

1. $\left[\underline{x}_{k}^{i j}, P_{k}^{i j}, L_{k}^{i j}\right]=\operatorname{filter}\left(\underline{x}_{k-1}^{i}, P_{k-1}^{i}, A_{k}, D_{k}, Q_{k}, H_{k}^{(j)}, R_{k}^{(j)}\right)$

In this function at each time $k$, first a priori estimate and its covariance matrix $\left(x_{k}^{-i j}\right.$ and $P_{k}^{-i j}$ ) are estimated from the previous time $k-1$. Then, by using the estimate $\underline{x}_{k}^{-i j}$ and observation $y_{k}$, the estimate is updated and the posterior estimate and its covariance $\left(\underline{x}_{k}^{i j}\right.$ and $\left.P_{k}^{i j}\right)$ are computed. In this function, $e_{k}$ is the innovation at time $k$. The likelihood $L_{k}^{i j}$ is the probability of the observation $y_{k}$ and can be calculated as a by-product of Kalman filter. Finally, $b_{k}$ is the Kalman gain matrix.

$$
\begin{aligned}
& \underline{x}_{k}^{-i j}=A_{k} \underline{x}_{k-1}^{i} \\
& P_{k}^{-i j}=A_{k} P_{k-1}^{i} A_{k}^{T}+D_{k} Q_{k} D_{k}^{T} \\
& e_{k}=y_{k}-H_{k}^{(j)} \underline{x}_{k}^{-i j} \\
& L_{k}^{i j}=\mathscr{N}\left(e_{k} ; 0, H_{k}^{(j)} P_{k}^{-i j} H_{k}^{(j)^{T}}+R_{k}^{(j)}\right) \\
& b_{k}=P_{k}^{-i j} H_{k}^{(j)^{T}}\left[H_{k}^{(j)} P_{k}^{-i j} H_{k}^{(j)^{T}}+R_{k}^{(j)}\right]^{(-1)} \\
& P_{k}^{i j}=\left[I-b_{k} H_{k}^{(j)}\right] P_{k}^{-i j} \\
& \underline{x}_{k}^{i j}=\underline{x}_{k}^{-i j}+b_{k}\left[y_{k}-H_{k}^{(j)} \underline{x}_{k}^{-i j}\right]
\end{aligned}
$$

2. $\left[K_{k}^{i j}, K_{k}^{j}, g_{k}^{i j}\right]=$ StatesProbability $\left(L_{k}^{i j}, z_{i j}, K_{k-1}^{i}\right)$ 
This function calculates the parameter $K_{k}^{j}$ for each mode $j$ at time $k$.

$$
\left\{\begin{array}{l}
i, j=1,2, \ldots, 7 \\
K_{k}^{i j}=\frac{L_{k}^{i j} z_{i j} K_{k-1}^{i}}{\sum_{i} \sum_{j} L_{k}^{i j} z_{i j} K_{k-1}^{i}} \\
K_{k}^{j}=\sum_{i} K_{k}^{i j} \\
g_{k}^{i j}=\frac{K_{k}^{i j}}{K_{k}^{j}}
\end{array}\right.
$$

375

3. $\left[\underline{x}_{k}^{j}, P_{k}^{j}\right]=$ Collapse $\left(\underline{x}_{k}^{i j}, P_{k}^{i j}, K_{k}^{j}, g_{k}^{i j}\right)$

This function approximates mixture of Gaussians as one Gaussian by moment matching.

$$
\left\{\begin{array}{l}
\underline{x}_{k}^{j}=\sum_{i} \underline{x}_{k}^{i j} g_{k}^{i j} \\
P_{k}^{j}=g_{k}^{i j}\left[P_{k}^{j}+\left(\underline{x}_{k}^{i j}-\underline{x}_{k}^{j}\right)\left(\underline{x}_{k}^{i j}-\underline{x}_{k}^{j}\right)^{T}\right]
\end{array}\right.
$$

